\title{
Preoperative Magnetic Resonance Imaging Diagnosis of Ovarian
}

\section{Torsion}

\author{
Yoshifumi Noda, ${ }^{1}$ Satoshi Goshima, ${ }^{1,}{ }^{*}$ Hiroshi Kawada, ${ }^{1}$ Nobuyuki Kawai, ${ }^{1}$ Hiromi Koyasu, ${ }^{1}$ and \\ Masayuki Matsuo ${ }^{1}$ \\ ${ }^{1}$ Department of Radiology, Gifu University, Gifu, Japan \\ "Corresponding author: Satoshi Goshima, Department of Radiology, Gifu University, 1-1 Yanagido, Gifu, Japan. Tel: +58-2306437, Fax: +58-2306440, E-mail: gossy@par.odn.ne.jp
} Received 2017 January 24; Revised 2017 May 30; Accepted 2017 October 09.

\begin{abstract}
Background: Magnetic resonance (MR) imaging, have become more frequently used in the diagnosis of ovarian torsion, particularly in subacute cases.

Objectives: To assess the characteristic findings and diagnostic performance of preoperative MR imaging for ovarian torsion.

Patients and Methods: Twenty-three patients (age range, 10 - 58 years; mean age, $28.4 \pm 12.5$ years) with suspected ovarian torsion underwent preoperative MR imaging. Fifteen patients exhibited ovarian torsion, whereas the remaining eight did not. Two radiologists evaluated the MR imaging data in relation to six previously-described characteristics associated with ovarian torsion, and the sensitivity, specificity, and accuracy of this approach was determined.

Results: Ovarian enlargement (maximum ovarian diameter $>4.0 \mathrm{~cm}$ ) and a twisted pedicle were significantly more common findings in patients with ovarian torsion compared with those without (regarding ovarian enlargement, $\mathrm{P}=0.032$ for both readers and considering twisted pedicle, $\mathrm{P}=$ 0.0094 for first reader and $\mathrm{P}=0.0013$ for second reader). The respective sensitivity, specificity, and accuracy of a twisted pedicle for the diagnosis of ovarian torsion were $73 \%, 88 \%$, and $78 \%$, respectively for reader 1 and $73 \%, 100 \%$, and $83 \%$, respectively for reader 2 .

Conclusion: Identifying a twisted pedicle in a patient is therefore, suggestive of ovarian torsion.
\end{abstract}

Keywords: Magnetic Resonance Imaging, Ovary, Ovarian Torsion, Twisted Pedicle

\section{Background}

Ovarian torsion is a serious but uncommon condition that can result in pain in the lower abdomen and pelvis in women. This condition is often difficult to distinguish from other differential diagnoses (1). Early diagnosis of ovarian torsion can help prevent irreversible ovarian damage and may allow conservative treatment. Urgent surgery is required in cases where complete ovarian torsion is suspected because of the risk of hemorrhagic infarction, a deleterious condition that can ultimately lead to peritonitis and even death (2). However, it is often difficult to make an accurate preoperative diagnosis of ovarian torsion. This is largely because this condition occasionally presents with symptoms that are associated with other causes of acute abdominal or pelvic pain.

Ultrasonography (US) is often used to detect ovarian torsion (3), although the validity of this particular technique is restricted by interoperator variability and the limited ability to diagnose other conditions associated with acute abdominal or pelvic pain. Over the recent years, alternative techniques, such as computer tomography (CT) and magnetic resonance (MR) imaging, have become more frequently accepted in the diagnosis of ovarian torsion, particularly in subacute cases (4-6). Existing literature indicate that a number of different characteristic findings such as ovarian enlargement, twisted pedicle, ovarian hemorrhage, abnormal ovarian enhancement, ascites, deviation of uterus to the twisted side, and thickening of the fallopian tube are associated with ovarian torsion (6-9). Our literature search did not unearth any previous studies that assessed the diagnostic performance of preoperative MR imaging for the diagnosis of ovarian torsion.

\section{Objectives}

The purpose of this study was to assess the characteristic findings and diagnostic performance of preoperative MR imaging for ovarian torsion.

\section{Patients and Methods}

\subsection{Patients}

Our institutional review board approved this retrospective study and the requirement for written informed consent was waived. From April 2006 to April 2014, 23 consecutive patients (age range, 10 - 58 years; mean age, 28.4 
\pm 12.5 years) with suspected ovarian torsion underwent preoperative MR imaging at our hospital. This group included 15 patients (age range, 10 - 58 years; mean age, 28.1 \pm 14.7 years) with and eight without surgically confirmed ovarian torsion (age range, 18 - 37 years; mean age, 28.9 \pm 7.7 years) (Figure 1 ). The interval between preoperative MR imaging and surgery ranged from 0 to 82 days (mean, $5.4 \pm 17.3$ days). The histopathologic diagnoses were corpus luteum cyst in five, normal ovary in five, mature cystic teratoma in four, serous cystadenoma in three, mucinous cystadenoma in two, fibroma in two, endometriotic cyst in one, and fallopian tube torsion in one patient. In detail, five were normal ovary, four were corpus luteum cyst, two were serous cystadenoma, two were mucinous cystadenoma, and two were fibroma in patients with ovarian torsion, and four were mature cystic teratoma, one was corpus luteum cyst, one was serous cystadenoma, one was endometriotic cyst, and one was fallopian tube torsion in patients without ovarian torsion.

\subsection{MR Imaging Protocol}

A 1.5-T MR system (Intera Achieva 1.5-T Pulsar; Philips Medical Systems, Netherlands) and a SENSE Torso coil was used to perform MR imaging. The basic MR imaging protocol consisted of the following imaging sequences: axial T1-weighted turbo spin-echo imaging (repetition time (TR)/echo time (TE), 759/15 msec; matrix, $416 \times 208$; field of view, $26 \times 26 \mathrm{~cm}$; parallel imaging factor, 2.3; 5-mm section thickness with a 2-mm intersection gap; acquisition time for 20 sections, 2 minutes); axial T2-weighted turbo spinecho imaging (TR/TE, 5,694/90 msec; matrix, $280 \times 240$; field of view, $26 \times 26 \mathrm{~cm}$; parallel imaging factor, 2; 5-mm section thickness with a 2-mm intersection gap; acquisition time for 20 sections, 2 minutes); sagittal T2-weighted turbo spin-echo imaging (TR/TE, 3,000/100 msec; matrix, $368 \times 258$; field of view, $28 \times 22 \mathrm{~cm}$; parallel imaging factor, 1.4; 5-mm section thickness with a 2-mm intersection gap; acquisition time for 20 sections, 2 minutes); coronal T2weighted turbo spin-echo imaging (TR/TE, 9,563/100 msec; matrix, $256 \times 179.2$; field of view, $40 \times 40 \mathrm{~cm}$; parallel imaging factor 2; 5-mm section thickness with a 1-mm intersection gap; acquisition time for 38 sections, 19 seconds); and diffusion-weighted single-shot turbo spin-echo echoplanar imaging (TR/TE, 5,008/60 msec; matrix, $112 \times 90$; field of view, $28 \times 28 \mathrm{~cm}$; parallel imaging factor, 2 ; b factors, 0 and $1,000 \mathrm{sec} / \mathrm{mm}^{2} ; 5-\mathrm{mm}$ section thickness with a 2-mm intersection gap; acquisition time for 20 sections, 2 minutes).

\subsection{Clinical Presentation}

Clinical information was obtained from the patients' medical records. Abdominal pain was defined as lower abdominal or flank pain. The onset of abdominal pain was defined as acute when it occurred within 24 hours before hospital admission, and gradual when it persisted for more than 24 hours before hospital admission. An elevated white blood cell (WBC) count was defined as > $12,000 / \mathrm{mm}^{3}$. Fever elevation was defined as a body temperature $>37.0^{\circ} \mathrm{C}(9)$.

\subsection{Image Analysis}

Image analysis was performed by two independent experienced radiologists who had no knowledge of the pathological and clinical information regarding the patients with 6 and 5 years of post-training experience in interpreting genitourinary images. The radiologists measured the maximum diameters of the affected ovaries and fallopian tubes on one of each of axial, sagittal, and coronal T2-weighted images. The radiologists assessed the presence of the following characteristic MR imaging findings that were associated with ovarian torsion (6-9): twisted pedicle (appearance of the fallopian tube in a helical configuration); ovarian hemorrhage; ascites; and deviation of the uterus to the side with the twist. Moreover, the radiologists assigned a confidence rating regarding the presence of MR imaging findings: 1 for definitely absent; 2 for probably absent; 3 for indeterminate; 4 for probably present; and 5 for definitely present.

\subsection{Statistical Analysis}

Statistical analysis was performed using commercially available software (MedCalc Software version 15.2.1, Ostend, Belgium). The incidence of WBC count and fever elevation and frequencies of MR imaging findings observed by each reader were compared between patients with and without ovarian torsion, and viable and nonviable ovaries using Fisher's exact test.

The Mann-Whitney U test was used to compare differences in the diameters of the affected ovaries and fallopian tubes in patients with and without ovarian torsion, and viable and nonviable ovaries. The intraclass correlation coefficient was calculated for each of the radiologist measurements in order to evaluate the significance of inter-observer differences. A P value of less than 0.05 was considered significant. The sensitivity for the detection of each MR imaging finding was defined by the number of patients assigned a rating of 4 or 5 among the total number of patients with ovarian torsion. Likewise, specificity was defined by the number of patients assigned a rating 1,2 , or 3 among the total number of patients without ovarian torsion. When assessing inter-observer variability regarding the confidence rating for each MR imaging finding and ovarian torsion, $\kappa$ statistics was used to 
23 consecutive patients (age range, $10-58$ years; mean, $28.4 \pm 12.5$ years)

with suspected ovarian torsion underwent preoperative MR imaging.

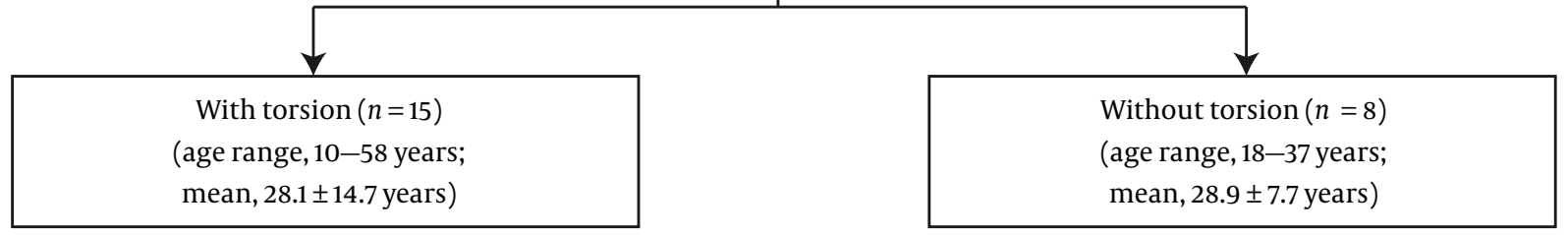

Figure 1. Flow diagram of study enrollment population

measure the degree of agreement between the two radiologists. A $\kappa$ value below 0.20 represents slight agreement, a value between 0.21 and 0.40 is considered as fair agreement, a value between 0.41 - 0.60 represents moderate agreement, a value between 0.61 and 0.80 represents substantial agreement, and a value greater than 0.81 represents almost perfect agreement. We conducted a post-hoc power analysis to assess our ability to detect differences between the two groups by using commercially available software (G*Power, version 3.1.2; University of Duesseldorf, Duesseldorf, Germany) with a given effect size of 0.50, a significance level of $5 \%$, and a total sample size of 23 patients.

\section{Results}

\subsection{Patients' Clinical Data}

All 23 patients suffered lower abdominal pain. The onset of pain was acute in six (40\%) and gradual in nine (60\%) of 15 patients with ovarian torsion, and was acute in three (38\%) and gradual in five (62\%) of eight patients without ovarian torsion. Three (20\%) of 15 patients with ovarian torsion and one (13\%) of eight patients without ovarian torsion had an elevated WBC count. Three (20\%) of 15 patients with ovarian torsion and one (13\%) of eight patients without ovarian torsion had a fever. We did not observe significant differences between patients with and without ovarian torsion in terms of frequency of acute abdominal pain onset $(\mathrm{P}=1.00)$, elevated $\mathrm{WBC}$ count $(\mathrm{P}=1.00)$, and fever $(\mathrm{P}$ $=1.00)$. Ovarian torsion occurred on the right side in eight (53\%) and the left side in seven patients (47\%). Among these 15 cases, five cases (33\%) were viable and 10 cases ( $67 \%$ ) were nonviable according to intra-operative findings.

\subsection{MR Imaging Findings}

The maximum diameters of the affected ovaries and fallopian tubes are summarized in Table 1. No significant differences were observed in terms of the maximum diameter of the affected ovary $(\mathrm{P}=0.44$ for reader 1 and $\mathrm{P}=0.52$ for reader 2$)$ or fallopian tube $(\mathrm{P}=0.56$ for reader 1 and $\mathrm{P}=0.42$ for reader 2 ) between patients with and without ovarian torsion (Figure 2). Similarly, no significant differences were observed in terms of the maximum diameter of the affected ovary $(\mathrm{P}=0.56$ for reader 1 and $\mathrm{P}=0.42$ for reader 2$)$ or fallopian tube $(\mathrm{P}=0.90$ for reader 1 and $\mathrm{P}=0.39$ for reader 2 ) between viable and nonviable ovaries. Interobserver variability was almost perfect (intraclass correlation coefficient range, 0.85 - 0.99).

Table 2 shows the frequencies of each MR imaging finding. A significant difference between patients with and without ovarian torsion is observed in terms of the frequency of ovarian enlargement $(\mathrm{P}=0.032$ for both readers $)$ and twisted pedicle $(\mathrm{P}=0.0094$ for reader 1 and $\mathrm{P}=0.0013$ for reader 2) (Figures 3 and 4). Ovarian enlargement was observed by both readers in all 15 patients with ovarian torsion. Similarly, both readers observed a twisted pedicle in 11 (73\%) patients with ovarian torsion. No significant differences were observed between patients with and without ovarian torsion in the following MR imaging findings (with versus without ovarian torsion for readers 1 and 2): ovarian hemorrhage ( $67 \%$ versus $63 \%, \mathrm{P}=1.00$ for both readers); ascites ( $93 \%$ versus $75 \%, \mathrm{P}=0.27$ for both readers); and deviation of the uterus to the side with the twist (20\% versus $12 \%, \mathrm{P}=1.00$ for reader $1 ; 33 \%$ versus $0 \%, \mathrm{P}=0.12$ for reader 2). No significant differences were observed in terms of MR imaging findings between viable and nonviable ovaries ( $\mathrm{P}$ $=0.40-1.00$ ).

We calculated the sensitivity, specificity, positive and negative predictive values, and accuracy of each MR imaging finding in the overall results of the two readers (Table 3). Both ovarian enlargement and ascites had high sensitivities (100\% and 93\%, respectively) but low specificities (38\% and 25\%, respectively). Although twisted pedicle had a 
Table 1. Maximum Diameter of the Affected Ovary and Fallopian Tube in Patients With and Without Ovarian Torsion, and Viable and Nonviable Ovaries ${ }^{\mathrm{a}}$

\begin{tabular}{|c|c|c|c|}
\hline Variables & Reader 1 & Reader 2 & ICC $(95 \% \mathrm{CI})$ \\
\hline \multicolumn{4}{|l|}{ Ovary } \\
\hline With ovarian torsion $(\mathrm{n}=15)$ & $79.9 \pm 42.9(44.0-211.0)$ & $80.5 \pm 50.8(46.0-205.0)$ & $0.99(0.97-0.99)$ \\
\hline Without ovarian torsion $(\mathrm{n}=8)$ & $66.4 \pm 34.1(28.0-116.0)$ & $67.3 \pm 34.1(31.0-117.0)$ & $0.99(0.99-1.00)$ \\
\hline Pvalue & 0.44 & 0.52 & - \\
\hline Viable $(\mathrm{n}=5)$ & $61.2 \pm 10.0(48.0-74.0)$ & $63.6 \pm 5.5(56.0-71.0)$ & $0.91(0.15-0.99)$ \\
\hline Nonviable $(n=10)$ & $89.2 \pm 50.3(44.0-211.0)$ & $89.0 \pm 50.2(46.0-205.0)$ & $0.99(0.96-0.99)$ \\
\hline Pvalue & 0.16 & 0.20 & - \\
\hline \multicolumn{4}{|l|}{ Fallopian tube } \\
\hline With ovarian torsion $(n=15)$ & $11.7 \pm 4.1(7.0-19.0)$ & $11.9 \pm 3.8(7.0-21.0)$ & $0.93(0.82-0.98)$ \\
\hline Without ovarian torsion $(\mathrm{n}=8)$ & $10.1 \pm 2.4(8.0-15.0)$ & $10.6 \pm 2.4(8.0-15.0)$ & $0.85(0.43-0.97)$ \\
\hline Pvalue & 0.56 & 0.42 & - \\
\hline Viable $(\mathrm{n}=5)$ & $12.6 \pm 5.9(8.0-19.0)$ & $13.8 \pm 5.4(9.0-21.0)$ & $0.97(0.77-0.99)$ \\
\hline Nonviable $(n=10)$ & $11.3 \pm 3.1(7.0-16.0)$ & $11.0 \pm 2.6(7.0-14.0)$ & $0.95(0.80-0.98)$ \\
\hline Pvalue & 0.90 & 0.39 & - \\
\hline
\end{tabular}

Abbreviations: CI, Confidence interval; ICC, Intraclass correlation coefficient.

${ }^{\mathrm{a}}$ Data are means \pm 1 standard deviation and data in parentheses are ranges.
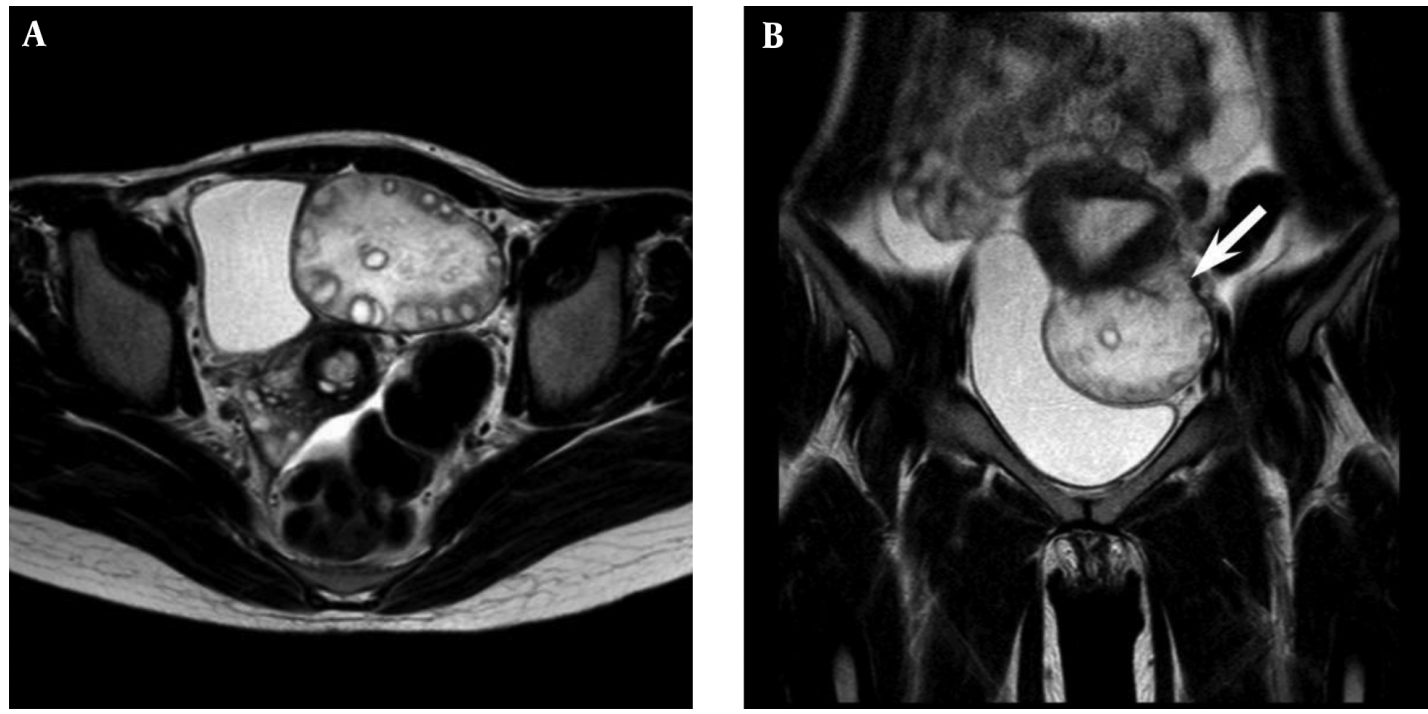

Figure 2. A 24-year-old woman with an enlarged, torsed left ovary. A, Axial T2-weighted image demonstrates an enlarged left ovary with a follicular central stroma and peripheral follicles. B, Coronal T2-weighted image demonstrates left fallopian tube thickening (arrow). Intraoperatively, the left ovary was found to be twisted by $540^{\circ}$.

slightly low sensitivity (73\%), this finding had a high specificity (94\%) and the accuracy (80\%) was high. The $\kappa$ values associated with the confidence ratings for the presence of each MR imaging finding and ovarian torsion were 0.79 0.89 , indicating substantial to almost perfect agreement between the two radiologists (Table 4). Post-hoc power analysis showed that we had $29 \%$ power to detect a differ- ence between the two groups.

\section{Discussion}

A twisted pedicle results in limitations in the outflow of both venous and lymphatic systems that can subsequently lead to arterial occlusion, ovarian ischemia, and ul- 
Table 2. MR Imaging Findings in Patients with and Without Ovarian Torsion, and Viable and Nonviable Ovaries ${ }^{\mathrm{a}}$

\begin{tabular}{|c|c|c|c|c|c|c|}
\hline \multirow[t]{2}{*}{ MR Imaging Findings } & \multicolumn{3}{|c|}{ Reader 1} & \multicolumn{3}{|c|}{ Reader 2} \\
\hline & $\begin{array}{l}\text { With Ovarian } \\
\text { Torsion, }(\mathbf{N}=15)\end{array}$ & $\begin{array}{l}\text { Without Ovarian } \\
\text { Torsion, }(\mathbf{N}=\mathbf{8})\end{array}$ & P Value & $\begin{array}{l}\text { With Ovarian } \\
\text { Torsion, }(\mathbf{N}=15)\end{array}$ & $\begin{array}{l}\text { Without Ovarian } \\
\text { Torsion, }(\mathbf{N}=\mathbf{8})\end{array}$ & P Value \\
\hline Ovarian enlargement & $15(100)$ & $5(63)$ & $0.032^{\mathrm{b}}$ & $15(100)$ & $5(63)$ & $0.032^{\mathrm{b}}$ \\
\hline Fallopian tube thickening & $8(53)$ & $4(50)$ & 1.00 & $11(73)$ & $5(63)$ & 1.00 \\
\hline Twisted pedicle & $11(73)$ & $1(12)$ & $0.0094^{\mathrm{b}}$ & $11(73)$ & $0(0)$ & $0.0013^{\mathrm{b}}$ \\
\hline Ovarian hemorrhage & $10(67)$ & $5(63)$ & 1.00 & $10(67)$ & $5(63)$ & 1.00 \\
\hline Ascites & $14(93)$ & $6(75)$ & 0.27 & $14(93)$ & $6(75)$ & 0.27 \\
\hline \multirow{2}{*}{$\begin{array}{l}\text { Deviation of uterus to the } \\
\text { sided of the twist }\end{array}$} & $3(20)$ & $1(12)$ & 1.00 & $5(33)$ & $0(0)$ & 0.12 \\
\hline & Viable, $(\mathbf{n}=\mathbf{5})$ & Nonviable, $(\mathbf{n}=10)$ & Pvalue & Viable, $(\mathbf{n}=5)$ & Nonviable, $(\mathrm{n}=10)$ & Pvalue \\
\hline Ovarian enlargement & $5(100)$ & $10(100)$ & 1.00 & $5(100)$ & $10(100)$ & 1.00 \\
\hline Fallopian tube thickening & $2(40)$ & $6(60)$ & 0.61 & $4(80)$ & $7(70)$ & 0.68 \\
\hline Twisted pedicle & $3(60)$ & $8(80)$ & 0.40 & $3(60)$ & $8(80)$ & 0.40 \\
\hline Ovarian hemorrhage & $3(60)$ & $7(70)$ & 0.70 & $4(40)$ & $6(60)$ & 0.60 \\
\hline Ascites & $5(100)$ & $9(90)$ & 1.00 & $5(100)$ & $9(90)$ & 1.00 \\
\hline $\begin{array}{l}\text { Deviation of uterus to the } \\
\text { sided of the twist }\end{array}$ & $0(0)$ & $3(30)$ & 0.51 & $1(20)$ & $4(40)$ & 0.60 \\
\hline
\end{tabular}

${ }^{\mathrm{a}}$ Values are expressed as No. (\%).

${ }^{\mathrm{b}} \mathrm{P}<0.05$, significant difference.
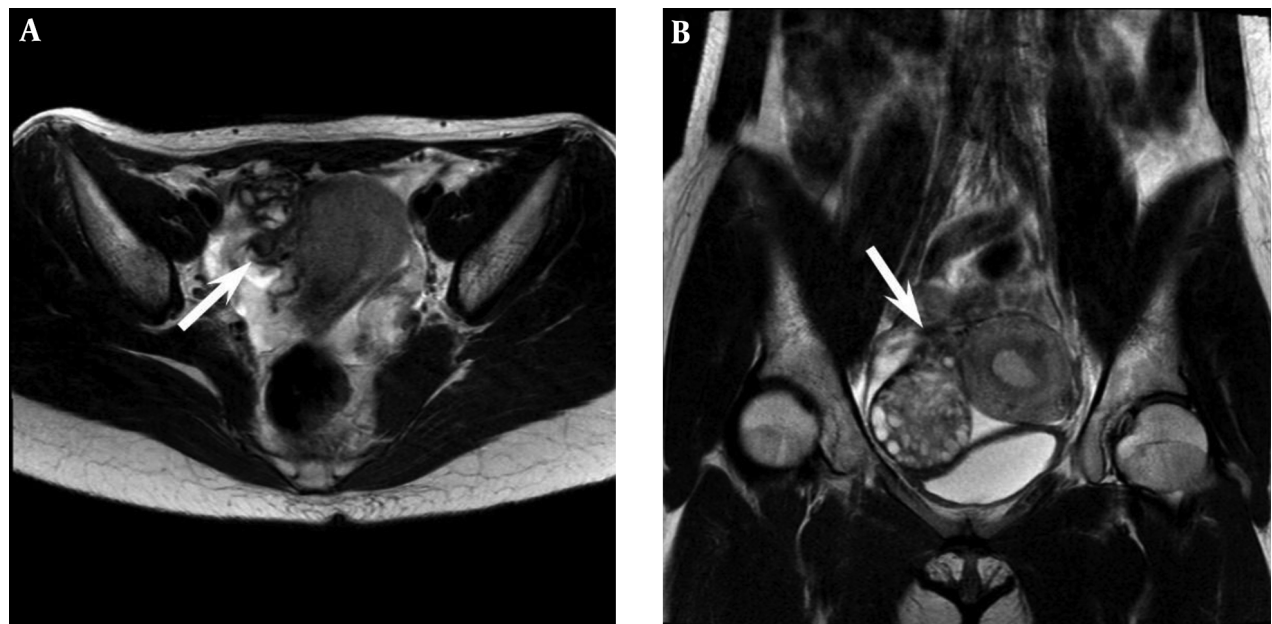

Figure 3. A 18-year-old woman with a torsed right ovarian fibroma and twisted pedicle. Axial (A) and coronal (B) T2-weighted images show a right ovarian mass connected to the broad ligament (arrow) with a helical swirling appearance. Intraoperatively, the right ovary was found to be twisted by $180^{\circ}$.

timately necrosis (10). In order to prevent these problems, a twisted pedicle requires urgent surgical intervention to remove the damaged tissue. However, physicians often fail to diagnose ovarian torsion because routine findings from physical examinations and laboratory tests generally are nonspecific (11).

In the present study, $60 \%$ of the patients with ovarian torsion did not experience an acute onset of abdominal pain. Furthermore, we were unable to identify a significant difference in the frequency of acute abdominal pain when compared between patients with and without ovarian torsion $(P=1.00)$. Although most laboratory findings are normal in patients with ovarian torsion, some studies have identified a minor degree of leukocytosis in $27 \%-50 \%$ of pa- 
Table 3. Sensitivity, Specificity, Positive and Negative Predictive Values, and Accuracy of Each MR Imaging Finding for the Diagnosis of Ovarian Torsion in the Overall Results of the Two Readers ${ }^{\mathrm{a}, \mathrm{b}}$

\begin{tabular}{|c|c|c|c|c|c|}
\hline MR Imaging Findings & Sensitivity & Specificity & PPV & NPV & Accuracy \\
\hline Ovarian enlargement & $100(30 / 30)$ & $38(6 / 16)$ & $75(30 / 40)$ & $100(6 / 6)$ & $78(36 / 46)$ \\
\hline Fallopian tube thickening & $63(19 / 30)$ & $44(7 / 16)$ & $68(19 / 28)$ & $39(7 / 18)$ & $35(16 / 46)$ \\
\hline Twisted pedicle & $73(22 / 30)$ & $94(15 / 16)$ & $96(22 / 23)$ & $35(15 / 23)$ & $80(37 / 46)$ \\
\hline Ovarian hemorrhage & $67(20 / 30)$ & $38(6 / 16)$ & $67(20 / 30)$ & $38(6 / 16)$ & $57(26 / 46)$ \\
\hline Deviation of uterus to the sided of the twist & $27(8 / 30)$ & $94(15 / 16)$ & $89(8 / 9)$ & $41(15 / 37)$ & $50(23 / 46)$ \\
\hline
\end{tabular}

Abbreviations: NPV, Negative predictive value; PPV, Positive predictive value.

${ }^{\mathrm{a}}$ Values are expressed as No. (\%).

${ }^{\mathrm{b}}$ Sensitivity, specificity, PPV, NPV, and accuracy values are percentages.

Table 4. Confidence Ratings of Each MR Imaging Finding and Ovarian Torsion ${ }^{\mathrm{a}}$

\begin{tabular}{|c|c|c|c|}
\hline MR Imaging Findings & Reader 1, Mean Confidence Rating & Reader 2, Mean Confidence Rating & $\kappa$ Value $(95 \% \mathrm{CI})$ \\
\hline Twisted pedicle & $3.8 \pm 1.0$ & $3.8 \pm 1.1$ & $0.81(-1.00-1.00)$ \\
\hline Ovarian hemorrhage & $3.7 \pm 1.3$ & $3.6 \pm 1.5$ & $0.80(0.66-0.95)$ \\
\hline Ascites & $4.4 \pm 1.5$ & $4.3 \pm 1.6$ & $0.89(-1.00-1.00)$ \\
\hline Deviation of uterus to the sided of the twist & $2.3 \pm 0.8$ & $2.4 \pm 0.9$ & $0.79(-1.00-1.00)$ \\
\hline Ovarian torsion & $3.9 \pm 0.9$ & $3.9 \pm 1.0$ & $0.83(-0.28-1.00)$ \\
\hline
\end{tabular}

Abbreviation: CI, Confidence interval.

${ }^{a}$ Values are expressed as mean $\pm S D$.

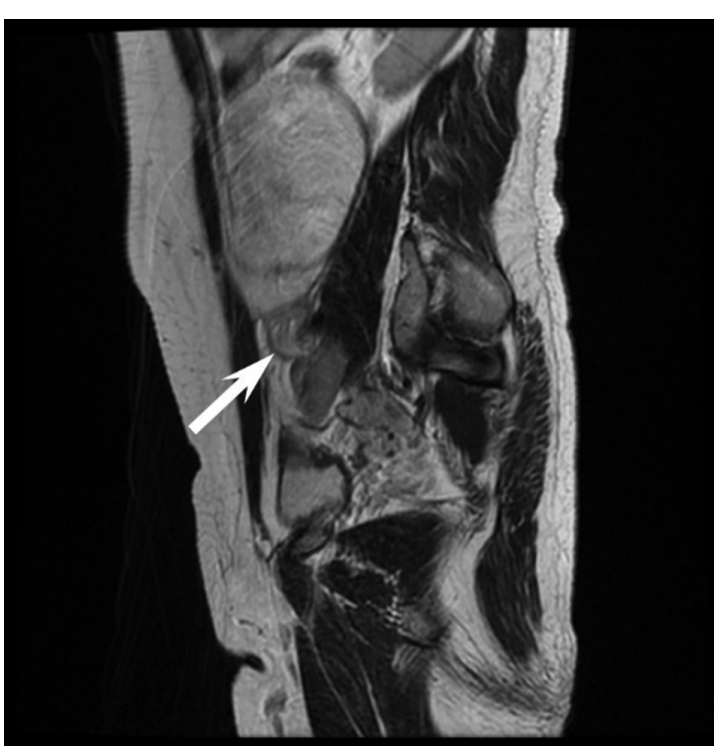

Figure 4. A 42-year-old woman with a torsed right ovarian serous cystadenoma and twisted pedicle. Sagittal T2-weighted image demonstrates a cystic right ovarian mass and twisted pedicle (arrow). Intraoperatively, the right ovary was twisted by $540^{\circ}$. tients $(12,13)$. In the present study, only $20 \%$ of the patients with ovarian torsion exhibited leukocytosis, and no statistical difference was found in the frequency of leukocytosis when compared between patients with and without ovarian torsion $(P=1.00)$. Previous literature has shown that ovarian torsion occurs more commonly on the right side of the body than the left, with an incidence ratio of approximately 3:2 (2). Here, however, the analysis observed no real difference (right side of the body, 53\% versus left side of the body, 47\%). It is highly apparent that the clinical of ovarian torsion is diagnostically of little use. Medical imaging is therefore, of much greater importance.

Previous studies have described a number of characteristic findings associated with ovarian torsion, which can all be identified using MR imaging. These include ovarian enlargement, twisted pedicle, ovarian hemorrhage, ascites, deviation of the uterus to the side with the twist, and fallopian tube thickening (6-9). Among these, the present results indicate that identifying a twisted pedicle was the most useful characteristic for the diagnosis of ovarian torsion. While a twisted pedicle can be difficult to detect, this represents an important pathognomonic feature, and is therefore, the most specific feature of ovarian torsion. However, studies have shown that less than one-third 
of ovarian torsion patients undergoing CT or MRI exhibit twisted pedicles $(9,14)$. In the present study, we observed a twisted pedicle in more than half of the patients with ovarian torsion. Furthermore, this finding exhibited the strongest diagnostic performance. Consequently, while a number of characteristic imaging findings have been associated with ovarian torsion, a twisted pedicle appears to represent the only finding directly indicative of ovarian torsion. All other imaging findings are indirect and exhibit large degrees of overlap with other differential diagnoses. It is well known that several other conditions resulting in enlarged ovaries can mimic ovarian torsion, such as serous cystadenoma, polycystic ovary, or ovarian hyperstimulation syndrome (7). Indeed, hemorrhagic ovarian cysts have been referred to as "the great imitator" because of their various appearances, which depends upon the age of the blood product at the time (15). Ovarian enlargement and twisted pedicle showed high accuracy for the diagnosis of ovarian torsion in this study. As written above, twisted pedicle is the only finding directly indicative of ovarian torsion and ovarian enlargement had a low specificity. Therefore, we believed that identifying the twisted pedicle was most important for the diagnosis of ovarian torsion in the clinical setting. Isolated fallopian tube torsion is extremely rare but should be considered in the differential diagnosis for ovarian torsion. Fallopian tube thickening has also been reported in the context of this latter condition (16).

Our study had the following limitations. First, this was a retrospective study with a relatively small cohort of patients at a single center, potentially resulting in selection bias because the post-hoc power analysis showed a power of $29 \%$. Further clinical studies are needed to validate our data. Second, we did not include contrast-enhanced or diffusion-weighted MR images because of the limited opportunity for examination time prior to surgery. However, we believe that gadolinium-enhanced or diffusionweighed MR imaging is likely to be very useful for evaluating ovarian enhancement, hemorrhagic infarction (17) or twisted pedicles.

In conclusion, our current study revealed that identification of a twisted pedicle represented a powerful and reliable diagnostic indicator with regard to ovarian torsion.

\section{Footnotes}

Authors' Contributions: All authors have equally contributed to this manuscript.

Financial Disclosure: Authors have no financial interests or conflicts.

Funding/Support: None declared.

\section{References}

1. Oltmann SC, Fischer A, Barber R, Huang R, Hicks B, Garcia N. Cannot exclude torsion-a 15-year review. J Pediatr Surg. 2009;44(6):1212-6. discussion 1217. doi:10.1016/j.jpedsurg.2009.02.028. [PubMed:19524743].

2. Nichols DH, Julian PJ. Torsion of the adnexa. Clin Obstet Gynecol. 1985;28(2):375-80. doi: 10.1097/00003081-198528020-00015. [PubMed: 4017325].

3. Swenson DW, Lourenco AP, Beaudoin FL, Grand DJ, Killelea AG, McGregor AJ. Ovarian torsion: Case-control study comparing the sensitivity and specificity of ultrasonography and computed tomography for diagnosis in the emergency department. Eur J Radiol. 2014;83(4):733-8. doi: 10.1016/j.ejrad.2014.01.001. [PubMed: 24480106].

4. Kimura I, Togashi K, Kawakami S, Takakura K, Mori T, Konishi J. Ovarian torsion: CT and MR imaging appearances. Radiology. 1994;190(2):33741. doi: 10.1148/radiology.190.2.8284378. [PubMed: 8284378].

5. Kim YH, Cho KS, Ha HK, Byun JY, Auh YH, Rhim HC, et al. CT features of torsion of benign cystic teratoma of the ovary.J Comput Assist Tomogr. 1999;23(6):923-8. doi: 10.1097/00004728-199911000-00016. [PubMed: 10589567].

6. Rha SE, Byun JY, Jung SE, Jung JI, Choi BG, Kim BS, et al. CT and MR imaging features of adnexal torsion. Radiographics. 2002;22(2):283-94. doi: 10.1148/radiographics.22.2.g02mr02283. [PubMed: 11896219].

7. Chang HC, BhattS, Dogra VS. Pearls and pitfalls in diagnosis of ovarian torsion. Radiographics. 2008;28(5):1355-68. doi: 10.1148/rg.285075130. [PubMed: 18794312].

8. Chiou SY, Lev-Toaff AS, Masuda E, Feld RI, Bergin D. Adnexal torsion: new clinical and imaging observations by sonography, computed tomography, and magnetic resonance imaging. J Ultrasound Med. 2007;26(10):1289-301. doi: 10.7863/jum.2007.26.10.1289. [PubMed: 17901133].

9. Hiller N, Appelbaum L, Simanovsky N, Lev-Sagi A, Aharoni D, Sella T. CT features of adnexal torsion. AJR Am J Roentgenol. 2007;189(1):124-9. doi: 10.2214/AJR.06.0073. [PubMed: 17579161].

10. Jain KA. Magnetic resonance imaging findings in ovarian torsion. Magn Reson Imaging.1995;13(1):111-3. doi:10.1016/0730-725X(94)00099O. [PubMed: 7898270].

11. Houry D, Abbott JT. Ovarian torsion: a fifteen-year review. Ann Emerg Med. 2001;38(2):156-9. doi: 10.1067/mem.2001.114303. [PubMed: 11468611].

12. Pansky M, Smorgick N, Herman A, Schneider D, Halperin R. Torsion of normal adnexa in postmenarchal women and risk of recurrence. Obstet Gynecol. 2007;109(2 Pt 1):355-9. doi: 10.1097/01.AOG.0000250969.15438.17. [PubMed:17267836].

13. Shadinger LL, Andreotti RF, Kurian RL. Preoperative sonographic and clinical characteristics as predictors of ovarian torsion. $\mathrm{J} \mathrm{Ul}$ trasound Med. 2008;27(1):7-13. doi: 10.7863/jum.2008.27.1.7. [PubMed: 18096725].

14. Comerci JJ, Licciardi F, Bergh PA, Gregori C, Breen JL. Mature cystic teratoma: a clinicopathologic evaluation of 517 cases and review of the literature. Obstet Gynecol. 1994;84(1):22-8. [PubMed: 8008317].

15. Yoffe N, Bronshtein M, Brandes J, Blumenfeld Z. Hemorrhagic ovarian cyst detection by transvaginal sonography: the great imitator. Gynecol Endocrinol. 1991;5(2):123-9. doi: 10.3109/09513599109028435. [PubMed: 1927577].

16. Gross M, Blumstein SL, Chow LC. Isolated fallopian tube torsion: a rare twist on a common theme. AJR Am J Roentgenol. 2005;185(6):1590-2. doi: 10.2214/AJR.04.1646. [PubMed: 16304018].

17. Kato H, Kanematsu M, Uchiyama M, Yano R, Furui T, Morishige K. Diffusion-weighted imaging of ovarian torsion: usefulness of apparent diffusion coefficient (ADC) values for the detection of hemorrhagic infarction. Magn Reson Med Sci. 2014;13(1):39-44. doi: 10.2463/mrms.2013-0039. [PubMed: 24492742]. 